cell line currently available which meets all the criteria proposed.

\section{Tense and Relaxed States}

\section{from our Molecular Biology Correspondent}

THe enzyme glutamine synthetase has been studied in Stadtman's laboratory and found to possess a number of curious properties. In particular, it seems that under standard assay conditions there is commonly a lag of some minutes before the enzyme reaction approaches maximum velocity. This phenomenon has now been explained (Kingdon et al., Biochemistry, ry, 2136; 1968) by an absolute requirement for certain divalent cations present in trace amounts in the buffer salts. Manganese and magnesium were found to be most effective cations causing activation. Calcium also functions, but at higher concentrations. The activation process is evidently eccompanied by a conformational change, which is reflected by the increased fluorescence of a bound dye. This effect is also reproduced in the presence of barium ions, which are ineffective in stimulating activity.

The nature of the interaction of the enzyme with the cations has been further studied by Shapiro and Ginsburg (ibid., 2153), who find that in the absence of the cations, the protein is in a less compact conformational state, called the "relaxed" form. The evidence for this "relaxed" form is a slightly diminished sedimentation coefficient $(19 \cdot 7 \mathrm{~S}$, as against $20 \cdot 3 \mathrm{~S})$, with no change in molecular weight, the appearance of an ultraviolet difference spectrum on activation, associated with decreased exposure of tyrosine and tryptophan residues to the solvent, and increased accessibility of thiol groups to specific reagents. Transition between the "relaxed" and "taut" states is an essentially reversible process, which can be driven in one direction by addition of EDTA, and in the other direction by the appropriate cations. The conformational rearrangement is quite slow, taking some twenty minutes to complete after addition of manganese ions, and follows what seem to be first-order kinetics. The relaxed form is more prone to dissociate into its subunits under marginal conditions, and is more labile towards denaturants. The enzyme contains covalently bound $5^{\prime}$-adenylic acid, and variations in the response of different preparations to metal ions have been correlated with differences in the number of such groups present per molecule.

The molecular weight of glutamine synthetase is about 600,000 , and electron microscopy (Valentine et al., ibid., 2143) shows that there are twelve subunits in the native enzyme, arranged in two hexagons, one lying on top of the other. All the indications are that the subunits are identical, and the molecule therefore contains a six-fold symmetry axis. Removal of cations produced no change in the overall shape of the protein, whereas dissociating reagents caused irregular breakdown of the geometry. When manganese or cobalt ions were added back to the relaxed enzyme, however, an extraordinary phenomenon occurred: the hexagonal structures associated to form long tubes. This effect was evidently favoured by low degrees of saturation with adenylic acid, for the tendency was suppressed in highly adenylated preparations. Valentine's work suggests that the prosthetic groups may be directly involved in the isologous set of sites between the hexagons. This type of indefinite association has been observed in one form or another elsewhere; it raises the general question (which arises also in the most general type of system-helical aggregates, such as bacterial flagella), what restrictions determine why the association ceases at a predetermined point (in the present case when two hexagons only have come together) in the native form of the protein and, by the same token, what allows the system to run riot under artificial conditions ?

\section{Chemicals from Petroleum}

\section{from a Correspondent}

THE eighty-seventh annual meeting of the Society of Chemical Industry in Edinburgh on July 16-20 followed the pattern which has been evolving in recent years. An important industrial and scientific subject was selected as the main theme for papers and discussion. Because the nearby town of Grangemouth has in the past twenty years rapidly grown to become one of Europe's largest petrochemical complexes, it was appropriate that the main theme of the meeting was the future of the petrochemical industry. Lectures at the symposium covered such topics as the source of petrochemicals, research for future development, future trends in petrochemical plant design, marketing and investment, industrial relations, future trends in plastics and the growth and performance of the British and North American chemical industries.

Petroleum is now the chief source of organic chemicals and it is estimated that the petroleum-based organic chemicals industry will increase in product volume by a factor of 15 to 20 by the end of the century. It is expected that more than 99 per cent of all primary organic chemicals will be derived from petroleum and natural gas by that time.

The chief building blocks for the petrochemical industry have been the supply of simple olefins, although petroleum is now taking over as a supplier of aromatic hydrocarbons. Ethylene plants are the focal points for the organio chemical industry and in the cracking of naphtha, 60 parts propylene, 35 parts $\mathrm{C}_{4}$ hydrocarbons, consisting of butadienes and the butenes, are formed as by-products for every 100 parts ethylene. These byproducts are further processed to chemical products: from ethylene come polyethylene, ethylene oxide, styrene and vinyl chloride; from propylene, polypropylene, acrylonitrile, cumene, acetone and butanol; from butadiene, synthetic rubber. The view was widely expressed that during the next ten years there will be a greater tendency to concentrate on existing chemicals, plastics and fibres and that there will be a decline in the number of new high profit products. For the future, it seems that in order to lessen capacity and marketing problems, the industry will tend to become more concentrated and there will be increasing cooperation between companies. As an illustration, frequent reference was made at the conference to the large number of refineries in the Common Market countries for the production of ethylene and other olefins which are linked by a comprehensive pipeline system to the oil ports on the North Sea and the Mediterranean.

The problems associated with the future design and operation of large petrochemical plants were discussed at length. It was considered that, to reach an economic size, the annual capacity today should be not less than 